Int. J. Morphol.,

30(2):374-378, 2012.

\title{
Ramo Superficial del Nervio Radial: Amplia Distribución en el Dorso de la Mano
}

\author{
Superficial Branch of the Radial Nerve: Large Distribution in the Dorsum of Hand \\ *Tiznado, G.; * Sousa-Rodrigues, C. \& ***:Olave, E.
}

TIZNADO, G.; SOUSA-RODRIGUES, C. \& OLAVE, E. Ramo superficial del nervio radial: Amplia distribución en el dorso de la mano. Int. J. Morphol., 30(2):374-378, 2012.

RESUMEN: La inervación sensitiva del dorso de la mano está dada generalmente por el ramo superficial del nervio radial y el ramo dorsal del nervio ulnar, donde el primero, alcanza de la zona lateral hasta la mitad radial del dedo anular y el segundo, la mitad ulnar de este dedo y también el dedo mínimo, no estando exenta de variaciones. Con el propósito de analizar la distribución del ramo superficial del nervio radial en el dorso de la mano, se estudiaron mediante disección 36 manos de cadáveres formolizados, 30 de individuos adultos brasileños y 6 de chilenos, encontrando en estas muestras, dos con una disposición especial en donde el ramo superficial del nervio radial fue el responsable de la totalidad de la inervación del dorso de la mano. En una de ellas, este ramo dio origen a tres ramos principales (R1, R2 y R3) los que se distribuyeron por el dorso de la mano con las respectivas divisiones para los dedos y en la otra muestra, este ramo emitió cuatro ramos principales (R1, R2, R3 y R4), distribuyéndose ampliamente por el dorso y dedos. El conocimiento de la disposición del ramo superficial del nervio radial y de sus ramos es de importancia para la anatomía quirúrgica de la región dorsal de la muñeca y mano, donde se realizan procedimientos que podrían poner en riesgo al nervio mencionado y sus ramos.

PALABRAS CLAVE: Anatomía, Mano; Nervio radial.

\section{INTRODUCCIÓN}

La inervación sensitiva del dorso de la mano está dada generalmente por el ramo superficial del nervio radial y el ramo dorsal del nervio ulnar, distribución en que el primero, abarca la zona desde el pulgar a la mitad radial del dedo anular y el segundo, la mitad ulnar de este dedo y también el dedo mínimo. Según Williams et al. (1995) en la región distal del antebrazo, el ramo superficial del nervio radial se curva en torno al lado lateral del radio, perfora la fascia profunda y se divide en cinco (a veces, en 4) nervios digitales dorsales. En el dorso de la mano, generalmente se comunica con los nervios cutáneos posterior y lateral del antebrazo. Los nervios digitales dorsales son 4 o 5 , donde el primero inerva el lado radial del pulgar; el segundo, el lado ulnar del pulgar; el tercero, el lado radial del indicador; el cuarto, los lados adyacentes del indicador y el medio y el quinto se comunica con un ramo dorsal del nervio ulnar e inerva los lados adyacentes del medio y anular.

Con respecto al ramo dorsal del nervio ulnar, estos autores señalaron que se origina en la parte distal del antebra- zo para dividirse luego en dos o frecuentemente en tres nervios digitales dorsales, uno inerva el lado ulnar del dedo mínimo; el segundo los lados adyacentes de los dedos mínimo y anular, mientras que el tercero, cuando presente, inerva los lados adyacentes de los dedos anular y medio, pero puede ser sustituído completa o parcialmente por un ramo del nervio radial, siempre comunicándose con él sobre el dorso de la mano.

Esta disposición es la que se encuentra con frecuencia y es la descrita en diferentes textos (Kaplan, 1965; Testut \& Latarjet, 1969; Healley \& Seybold, 1972; Sunderland, 1985: Moore \& Dalley, 2002), sin embargo, como las variaciones están presentes en el cuerpo humano, se puede observar como el ramo superficial del nervio radial o el ramo dorsal del nervio ulnar pueden ocupar áreas correspondientes al otro nervio parcial o totalmente o superponerse el uno con el otro (Sunderland; Williams et al.; Tountas \& Bergman, 1993), inclusive estos últimos autores han señalado que se han descrito casos en que el nervio radial ha estado ausente y ha sido reemplazado por ramos del nervio antebraquial

\footnotetext{
* Universidad San Sebastián, Sede Osorno, Chile; Programa de Magíster en Ciencias, mención, Morfología, Universidad de La Frontera, Chile.

** Universidad Federal de Alagoas y Universidad Provincial de Ciencias de La Salud de Alagoas, Maceió, Brasil.

${ }^{* * *}$ Universidad de La Frontera, Temuco, Chile.
} 
cutáneo lateral (para el pulgar) y el resto por ramos del nervio ulnar, como también el ramo superficial del nervio radial puede enviar ramos a los dedos anular y mínimo, disposición también descrita por Williams et al.

Debido a la importancia de estos nervios sensitivos, sobretodo por los procedimientos quirúrgicos que se realizan en la muñeca y en el dorso del carpo, intervenciones que los pueden poner en riesgo, el interés del presente trabajo fue aportar al conocimiento de la inervación sensitiva del dorso de la mano, mostrando una especial disposición del ramo superficial del nervio radial en el dorso de la mano, cuando éste es responsable de la inervación total de esta región.

\section{MATERIAL Y MÉTODO}

Se utilizaron 30 manos de cadáveres de individuos adultos, brasileños, fijados en solución acuosa de formaldehido al $10 \%$ y 6 manos formolizadas, de cadáveres de individuos adultos, de nacionalidad chilena. Los primeros pertenecen a los Laboratorios de Anatomía de la Universidade de Ciencias da Saude, Maceió, Alagoas, Brasil y los segundos a los Laboratorios de Anatomía de la Facultad de Medicina de la Universidad de La Frontera, Temuco, Chile.

Se realizó disección del dorso de cada una de la manos, poniendo en evidencia el ramo superficial del nervio radial y su división en esta región. De los casos estudiados, dos muestras presentaron inervación total del dorso de la mano por parte del ramo superficial del nervio radial, una del grupo brasileño y la otra del grupo chileno. Los ramos derivados del ramo superficial del nervio radial fueron nominados de lateral hacia medial como R1, R2, R3 y R4. Las disecciones fueron fotografiadas y esquematizadas.

\section{RESULTADOS}

Del total de las manos estudiadas se encontraron dos $(5,6 \%)$ con la disposición en que el ramo superficial del nervio radial fue responsable de la totalidad de la inervación sensitiva en esta región, distribuyéndose enteramente en el dorso de la mano.

En la muestra 1 (Fig. 1), mano izquierda, proximal a la articulación de la muñeca, el ramo superficial del nervio radial dio origen a un ramo (R1) que tenía un trayecto sobre el dorso de la parte lateral del carpo y la zona del primer hueso metacarpiano. Distal a su origen $(30 \mathrm{~mm})$, este ramo se dividió en dos ramos secundarios (lateral y medial), de los cuales, el lateral continuaba directo sobre el dorso del primer metacarpiano en dirección a la parte lateral del dorso del pulgar y el medial, se dividió a 13,8 mm de su origen en dos ramos menores que iban, uno hacia la parte medial del dorso del pulgar y el otro a la parte lateral del indicador.

Después de emitir el R1, el ramo superficial del nervio radial se dividió en dos ramos, R2 y R3. El R2, después de su origen se bifurcó en un ramo lateral, que se dirigía hacia la zona del segundo espacio interóseo donde dio origen al ramo digital propio medial del dedo indicador y al ramo digital palmar propio lateral del dedo medio, $\mathrm{y}$, en un ramo medial, que cruzó oblicuamente el dorso de la mano para alcanzar la zona del tercer espacio interóseo donde se dividió en dos nervios digitales dorsales propios para el dedo

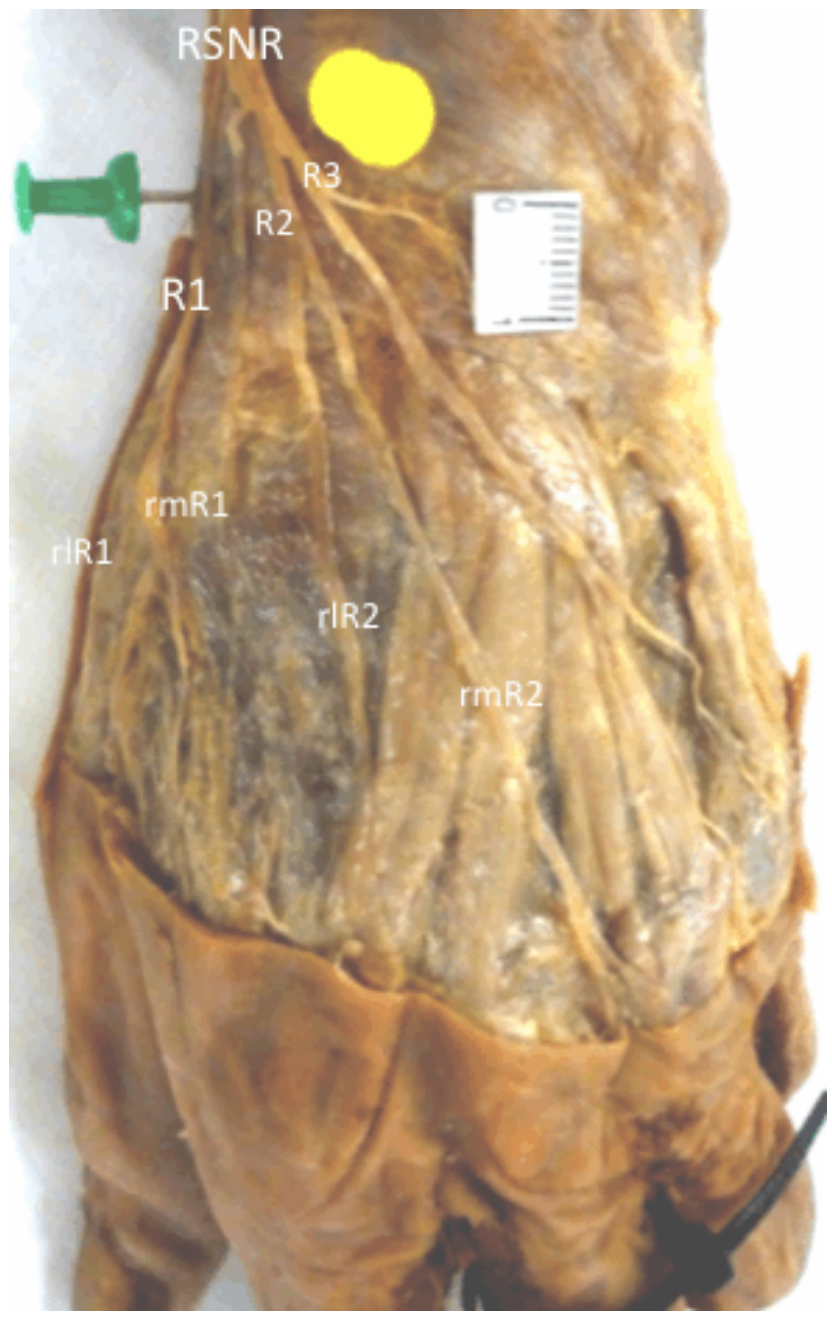

Fig. 1. Ramo superficial del nervio radial (RSNR); ramo principal 1 (R1); ramo principal 2 (R2); ramo principal 3 (R3); ramo lateral del ramo 1 (rlR1); ramo medial del ramo 1 (rmR1); ramo lateral del ramo 2 (rlR2); ramo medial del ramo 2 (rmR2). 
medio y el dedo anular. El R3 emitió un primer ramo, menor, que va hacia la zona de la articulación de la muñeca, para luego continuar oblicuamente sobre el dorso de la mano, dividiéndose a $45 \mathrm{~mm}$ después de su origen, en tres ramos, dos de los cuales, laterales, van hacia la zona del cuarto espacio interóseo para los dedos anular y mínimo, y, un ramo que va hacia la zona medial del dedo mínimo.

En la muestra 2 (Fig. 2), mano derecha, el ramo superficial del nervio radial dio origen, proximal a la articulación de la muñeca, a cuatro ramos principales (R1, R2, R3 y $\mathrm{R} 4)$, donde el R1 se dirige hacia la parte lateral del dorso del pulgar; el ramo R2, medial a este último, a $60 \mathrm{~mm}$ de su origen se dividió en dos ramos secundarios, que a su vez se subdividieron nuevamente en dos ramos menores cada uno, los que fueron en dirección a la parte medial del dorso del pulgar y parte lateral del dedo indicador.

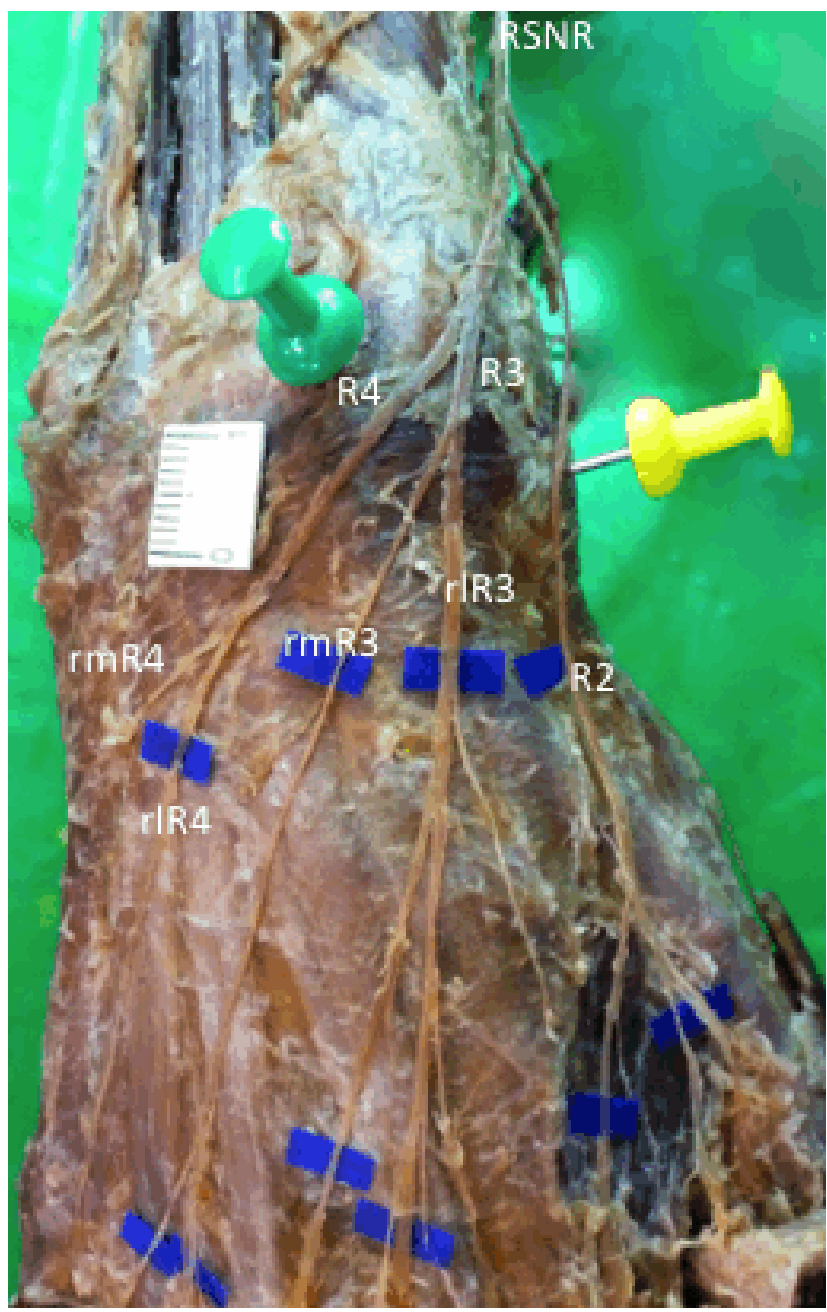

Fig. 2. Ramo superficial del nervio radial (RSNR); ramo principal 2 (R2); ramo principal 3 (R3); ramo principal 4 (R4); ramo lateral del ramo 3 (rlR3); ramo medial del ramo 3 (rmR3); ramo lateral del ramo 4 (rlR4); ramo medial del ramo 4 (rmR4).
Distalmente, el ramo superficial del nervio radial, $11,4 \mathrm{~mm}$ distal al R2, se dividió en dos ramos terminales (R3 y R4). El R3, a 6,4 mm distal a su origen emitió un ramo menor, medial, que se dirigía oblicuamente por el dorso de la mano, hacia la zona del tercer espacio interóseo y, donde cerca de la articulación metacarpofalángica se dividió en dos ramos menores que se dirigían hacia la parte medial del dedo medio y la parte lateral del dedo anular.

Luego de otorgar este ramo, $20 \mathrm{~mm}$ distal a este último, el R3 emite un ramo hacia lateral que va en dirección hacia el dorso del dedo indicador; el mismo R3 luego de un trayecto de $10,7 \mathrm{~mm}$, se divide en dos ramos, lateral y medial. El ramo lateral de R3 se dirige hacia la zona del dedo indicador, subdividiéndose en dos ramos menores, uno lateral que alcanza la parte dorsal del dedo indicador y uno medial, más grueso, que se subdivide en tres ramos, uno de los cuales, el lateral, acompaña al ramo dirigido hacia el indicador y los otros dos van hacia la parte medial del dedo indicador. El ramo medial se dirige oblicuamente hacia la zona de la parte lateral del dedo medio. El ramo medial del R3 se dirige hacia la parte medial del dedo medio y lateral del dedo anular.

El R4 cruza oblicuamente el dorso de la articulación de la muñeca y el carpo, y a 34,3 mm después de su origen se divide en dos ramos menores, uno medial que va hacia la zona del quinto hueso metacarpiano para alcanzar la parte medial del dedo mínimo y uno lateral, que transcurre por sobre la zona del cuarto espacio interóseo para alcanzar la parte medial del dedo anular y la parte lateral del dedo mínimo.

\section{DISCUSIÓN}

La clásica descripción sobre la inervación sensitiva del dorso de la mano, atribuída a ramos procedentes de los nervios radial y ulnar, en que el primero tiene como límite aproximado de inervación la zona desde el pulgar hasta la parte lateral del dedo medio y por comunicación con ramos del nervio ulnar hasta las apartes adyacentes del dedo medio y anular (Williams et al.), puede presentar variaciones de invasión de una zona por parte de cualquiera de los dos nervios. Sin embargo, son pocos los casos en que se puede observar la inervación total del dorso por parte de alguno de los nervios mencionados.

Autores como Tountas \& Bergman describen que el ramo superficial del nervio radial inerva el dorso del pulgar, ambos lados del dedo indicador y el lado radial del dedo medio, indicando además que el nervio radial puede enviar 
ramos dorsales digitales al dedo mínimo y al lado ulnar del dedo anular, en vez del nervio ulnar. Otras variaciones han sido mencionadas por estos mismos autores, como la que el nervio radial puede inervar el dedo medio e indicador a través de ramos emitidos por su ramo interóseo posterior. También señalaron que el nervio radial puede inervar enteramente el dorso de la mano, incluyendo la cara dorsal de todos los dedos, disposición que también es señalada por Williams et al. y que corresponde a los dos casos presentados en este artículo.

El conocimiento de la disposición del ramo superficial del nervio radial y de sus ramos derivados es de importancia para la anatomía quirúrgica de la región dorsal de la muñeca y mano, donde se realizan procedimientos de artroscopías aplicadas a las articulaciones trapeciometacarpiana y metacarpofalángica del pulgar, particularmente en las trapecioctomías parciales o totales, tratamientos de lesiones ligamentosas de articulaciones metacarpofalángicas y sinovectomías, procedimientos que podrían poner en riesgo al nervio mencionado y sus ramos (Ropars et al., 2010). También estos ramos pueden ser lesionados en procedimientos de punciones de la vena cefálica (Boeson et al., 2000).

De acuerdo a una investigación realizada por Ikiz \& Üçerler (2004) en el 89,55 \% de sus casos (48 manos) los ramos derivados del ramo superficial del nervio radial, alcanzaron, uno el más medial la parte dorsal del dedo indicador, el segundo, alcanzó el primer espacio interóseo y el tercero la parte lateral del pulgar. En 8,33\%, estos autores observaron que el ramo superficial del nervio radial, una vez subcutáneo, en la parte distal del antebrazo, continuó distal y separado en tres ramos sin ninguna bifurcación.

El primer tipo mencionado por Ikiz \& Üçeler fue observado por Huanmanop et al. (2007) en $61,1 \%$ de 22 miembros superiores de sexo masculino y en $67,4 \%$ de 29 miembros femeninos estudiados. Por su parte, Loukas et al.
(2008) investigando en 120 muestras los ramos comunicantes entre los ramos superficial del nervio radial y el ramo dorsal del nervio ulnar en el dorso de la mano, no encontraron en el dorso ningún caso con inervación total por parte del ramo superficial del nervio radial. En otro estudio realizado por Chen et al. (2010) en 19 de 20 muestras el ramo superficial del nervio radial se dividió en un nervio digital dorsal propio radial para el dedo pulgar, en el primer nervio digital dorsal común y en el segundo nervio digital dorsal común. Recientemente Korcek \& Wongworawat (2011) en 40 muestras no encontraron ni ocupación ni sobreposición del ramo superficial del nervio radial en el área correspondiente al ramo dorsal del nervio ulnar.

Los autores mencionados no encontraron una disposición como la informada en este trabajo, por lo que es de interés, tener presente su posible presencia durante los procedimientos quirúrgicos realizados en esta región de la mano.

Por su parte, Vergara-Amador \& Nieto (2010) determinaron en 25 muestras que a nivel del proceso estiloides del radio, el ramo superficial del nervio radial se dividía en dos ramos en 16 casos (64\%), en tres ramos en 7 (28\%), en cuatro ramos en 1 caso y en cinco en 1 caso. Estos autores encontraron que en uno de sus casos (4\%) hubo una disposición en que sus ramos alcanzaron una inervación dorsal hasta el lado radial del dedo mínimo, no inervando el lado ulnar de este dedo. La disposición mostrada en el presente artículo es similar a la mencionada por estos últimos autores.

Como se puede apreciar, los padrones descritos por diferentes autores sobre la inervación del dorso de la mano y los dedos, involucran a los ramos superficial del nervio radial y ramo dorsal del nervio ulnar, no estando exentos de variaciones, las que ocasionalmente se presentan como disposiciones que abarcan la totalidad del dorso de la mano por parte exclusiva de uno de los nervios mencionados, como los casos presentados en este artículo.

TIZNADO, G.; SOUSA-RODRIGUES, C. \& OLAVE, E. Superficial branch of the radial nerve: Large distribution in the dorsum of hand. Int. J. Morphol., 30(2):374-378, 2012.

SUMMARY: The sensory innervation of the dorsum of the hand is usually given by the superficial branch of radial nerve and the dorsal branch of ulnar nerve, where the first, reaches the lateral zone of the dorsum to the radial half of ring finger and the second, the ulnar half of this finger and the little toe, not being free from variations. In order to analyze the distribution of the superficial branch of radial nerve in the dorsum of the hand, we studied by dissection of cadaver specimens, 36 hands, 30 adult Brazilians and 6 Chileans, finding in these samples, two with a special disposition where the superficial branch of radial nerve was responsible for all of the innervation of the dorsum of the hand. The main branches were nominated from lateral to medial zone. In one of them, this branch gave rise to three main branches (R1, R2 and R3) which were distributed by the dorsum of the hand with the respective divisions for the fingers and the other case, this branch originated four main branches (R1, R2, R3 and R4), distributed widely in the dorsum and fingers. Knowledge of the arrangement of the superficial branch of radial nerve and its branches is of importance to the surgical anatomy of the dorsal region of the wrist and hand, where there are procedures that could jeopardize the nerve and its branches.

KEY WORDS. Anatomy; Hand; Radial nerve. 


\section{REFERENCIAS BIBLIOGRAFICAS}

Boeson, M.B.; Hranchook, A. \& Stoller, J. Peripheral nerve injury from intravenous cannulation: a case report. AANA. J., 68: 53-7, 2000.

Chen, Y.; Zheng, X.; Wang, J.; Zhu, Y. \& Zhan, Ch. Reliable techniques to avoid damaging the superficial radial nerve due to percutaneous Kirschner wire fixation to the distal radius fracture through the radial styloid process. Surg. Radiol. Anat., 32:711-7, 2010.

Healey, J. \& Seybold, W . Anatomía Clínica. 1ª Ed. D.F., Mexico, Interamericana, 1972.

Huanmanop, T.; Agthong, S.; Luengchawapong, K.; Sasiwongpakdee, T.; Burapasomboon, P. \& Chentanez, V. Anatomic characteristic and surgical implications of the superficial radial nerve. J. Med. Assoc. Thai., 90(7):1423-9, 2007.

Ikiz, Z. A. A. \& Üçerler, H. Anatomic characteristic and clinical importance of the superficial branch of the radial nerve. Surg. Radiol. Anat., 26:453-8, 2004.

Kaplan, E. B. Functional and Surgical Anatomy of the Hand. $2^{\text {nd }}$ Ed. Philadelphia, Lippincott Co., 1965.

Korcek, L. \& Wongworawat, M. Evaluation of the safe zone for percutaneous Kirschner-wire placement in the distal radius: cadaveric study. Clin. Anat., 24:1005-9, 2011.

Loukas, M.; Louis Jr., R.G.; Wartmann, C.T.; Tubbs, R.S.; TuranOzdemir, S. \& Kramer, J. The clinical anatomy of the communications between the radial and ulnar nerves on the dorsal surface of the hand. Surg. Radiol. Anat., 30: 85-90, 2008.

Moore, K. L. \& Dalley, A. F. Anatomía con orientación Clínica. $4^{\text {th }}$ Ed. Buenos Aires, Panamericana, 2002.

Ropars, M.; Fontaine, I.; Morandi, X.; Berton, E.; Kaila, R. \& Darnault, P. Preserving the superficial branch of the radial nerve during carpometacarpal and metacarpophalangeal joint arthroscopy: an anatomical study. Surg. Radiol. Anat., 32: 2716, 2010.

Tountas, Ch. \& Bergman, R. Anatomic variations of the upper extremity. New York, Churchill Livingstone, 1993.

Sunderland, S. Nervios Periféricos y sus lesiones. Barcelona, Salvat, 1985.

Testut, L. \& Latarjet, A. Tratado de Anatomía Humana. Barcelona, Salvat, 1969. V. 3.

Vergara-Amador, E. \& Nieto, J.L. Estudio anatómico de la rama superficial del nervio radial. Implicaciones quirúrgicas. Rev. Fac. Med., 58(3):214-20, 2010.
Williams, R L.; Warwick, R.; Dyson, M. \& Bannister, L. H. Gray Anatomía. 37a Ed. Rio de Janeiro, Guanabara-Koogan, 1995. V. 2.

Dirección para correspondencia:

Prof. Dr. Enrique Olave

Facultad de Medicina

Universidad de La Frontera

casilla 54-D

Temuco

CHILE

Email: eolave@ufro.cl

Recibido : 12-01-2012

Aceptado: 05-02-2012 